\title{
ON THE BEHAVIOR AT INFINITY OF AN INTEGRABLE FUNCTION
}

\author{
EMMANUEL LESIGNE
}

We denote by $x$ a real variable and by $n$ a positive integer variable. The reference measure on the real line $\mathbb{R}$ is the Lebesgue measure. In this note we will use only basic properties of the Lebesgue measure and integral on $\mathbb{R}$.

It is well known that the fact that a function tends to zero at infinity is a condition neither necessary nor sufficient for this function to be integrable. However, we have the following result.

Theorem 1. Let $f$ be an integrable function on the real line $\mathbb{R}$. For almost all $x \in \mathbb{R}$, we have

$$
\lim _{n \rightarrow \infty} f(n x)=0 .
$$

Remark 1. It is too much hope in Theorem 1 for a result for all $x$ because we consider an integrable function $f$, which can take arbitrary values on a set of zero measure. Even if we consider only continuous functions, the result does not hold for all $x$. Indeed a classical result, using a Baire category argument, tells us that if $f$ is a continuous function on $\mathbb{R}$ such that for all nonzero $x, \lim _{n \rightarrow \infty} f(n x)=0$, then $\lim _{x \rightarrow \pm \infty} f(x)=0$. Thus for a continuous integrable function $f$ which does not tend to zero at infinity, property (11) is true for almost all $x$ and not for all $x$.

Remark 2. Let $f$ be an integrable and nonnegative function on $\mathbb{R}$. We have $\int f(n x) \mathrm{d} x=\frac{1}{n} \int f(x) \mathrm{d} x$. Hence for any nonnegative real sequence $\left(\varepsilon_{n}\right)$ such that $\sum_{n} \epsilon_{n} / n<+\infty$, we have

$$
\sum_{n} \int \varepsilon_{n} f(n x) \mathrm{d} x<+\infty
$$

and the monotone convergence theorem (or Fubini's theorem) ensures that the function $x \mapsto \sum_{n} \varepsilon_{n} f(n x)$ is integrable, hence almost everywhere finite. In particular, for almost all $x$, we have $\lim _{n \rightarrow \infty} \varepsilon_{n} f(n x)=0$. This argument is not sufficient to prove Theorem it.

Now we will state that, in a sense, Theorem 1 gives an optimal result. The strength of the following theorem lies in the fact that the sequence $\left(a_{n}\right)$ can tend to infinity arbitrarily slowly.

Theorem 2. Let $\left(a_{n}\right)$ be a real sequence which tends to $+\infty$. There exists a continuous and integrable function $f$ on $\mathbb{R}$ such that, for almost all $x$,

$$
\limsup _{n \rightarrow \infty} a_{n} f(n x)=+\infty
$$


Moreover, there exists an integrable function $f$ on $\mathbb{R}$ such that, for all $x$,

$$
\limsup _{n \rightarrow \infty} a_{n} f(n x)=+\infty \text {. }
$$

Question. Under the hypothesis of Theorem 2, does there exist a continuous and integrable $f$ such that, for all $x, \limsup _{n \rightarrow \infty} a_{n} f(n x)=+\infty$ ?

We do not know the answer to this question, and we propose it to the reader. However, the next remark shows that the answer is positive under a slightly more demanding hypothesis.

Remark 3. If the sequence $\left(a_{n}\right)$ is nondecreasing and satisfies $\sum_{n} \frac{1}{n a_{n}}<+\infty$, then there exists a continuous and integrable function $f$ on $\mathbb{R}$ such that for all $x$, $\lim \sup _{n \rightarrow \infty} a_{n} f(n x)=+\infty$.

Remark 4. In Theorem 2 we cannot replace the hypothesis $\lim _{n} a_{n}=+\infty$ by $\lim \sup _{n} a_{n}=+\infty$. Indeed, by a simple change of variable we can deduce from Theorem 1 the following result: for all integrable functions $f$ on $\mathbb{R}$,

$$
\lim _{n \rightarrow \infty} n f\left(n^{2} x\right)=0 \text { for almost all } x .
$$

(Apply Theorem 1 to the function $x \mapsto x f\left(x^{2}\right)$.) Thus the conclusion of Theorem 2 is false for the sequence $\left(a_{n}\right)$ defined by

$$
a_{n}= \begin{cases}\sqrt{n} & \text { if } n \text { is a square of integer, } \\ 0 & \text { if not. }\end{cases}
$$

In the remainder of this note, we give proofs of the two theorems and of Remark 3.

Proof of Theorem $⿴$. The function $f$ is integrable on $\mathbb{R}$. Let us fix $\varepsilon>0$ and denote by $E$ the set of points $x>0$ such that $|f(x)| \geq \varepsilon$. We know that $E$ has finite measure. We are going to show that, for almost all $x \in[0,1]$, we have $n x \in E$ for only finitely many $n$ 's. (If $A$ is a measurable subset of $\mathbb{R}$, we denote by $|A|$ its Lebesgue measure.)

For each integer $m \geq 1$, let $E_{m}:=E \cap(m-1, m]$. Let us fix $a \in(0,1)$. For each integer $n \geq 1$, we consider the set

$$
F_{n}:=\left(\frac{1}{n} E\right) \cap[a, 1)=\left(\frac{1}{n} \bigcup_{m \geq 1} E_{m}\right) \cap[a, 1)=\frac{1}{n} \bigcup_{m \geq 1}\left(E_{m} \cap[n a, n)\right) .
$$

We have

$$
\sum_{n=1}^{+\infty}\left|F_{n}\right|=\sum_{n=1}^{+\infty} \sum_{m=1}^{+\infty} \frac{1}{n}\left|E_{m} \cap[n a, n)\right| .
$$

In this doubly indexed sum of positive numbers, we can invert the order of summation. Moreover, noticing that $E_{m} \cap[n a, n)=\emptyset$ if $n>m / a$ or $n \leq m-1$, we obtain

$$
\sum_{n=1}^{+\infty}\left|F_{n}\right|=\sum_{m=1}^{+\infty} \sum_{n=m}^{[m / a]} \frac{1}{n}\left|E_{m} \cap[n a, n)\right| \leq \sum_{m=1}^{+\infty}\left|E_{m}\right| \sum_{n=m}^{[m / a]} \frac{1}{n}
$$


By comparison of the discrete sum with an integral, we see that, for all $m \geq 1$, $\sum_{n=m}^{[m / a]} \frac{1}{n} \leq(1-\ln a)$. Thus we have

$$
\sum_{n=1}^{+\infty}\left|F_{n}\right| \leq(1-\ln a) \sum_{m=1}^{+\infty}\left|E_{m}\right|=(1-\ln a)|E|<+\infty .
$$

This implies that almost every $x$ belongs to only finitely many sets $F_{n}$. (This statement is the Borel-Cantelli lemma, which has a one line proof :

$\sum \mathbb{1}_{F_{n}}<+\infty$ almost everywhere since

$$
\left.\int \sum \mathbb{1}_{F_{n}}(x) \mathrm{d} x=\sum \int \mathbb{1}_{F_{n}}(x)<+\infty .\right)
$$

Returning to the definition of $F_{n}$, we conclude that, for almost all $x \in[a, 1]$, for all large enough $n, x \notin F_{n}$, i.e. $n x \notin E$. Since $a$ is arbitrary, we have in fact: for almost all $x \in[0,1]$, for all large enough $n, n x \notin E$.

We have proved that, for all $\varepsilon>0$, for almost all $x \in[0,1]$, for all large enough $n,|f(n x)| \leq \varepsilon$. Since we have to consider only countably many $\varepsilon$ 's, we can invert for all $\varepsilon>0$ and for almost all $x \in[0,1]$. We conclude that, for almost all $x \in[0,1]$, $\lim _{n \rightarrow \infty} f(n x)=0$. It is immediate, by a linear change of variable (for example), that this result extends to almost all $x \in \mathbb{R}$.

Proof of Theorem 6. We will utilize the following theorem, a fundamental result in the metric theory of Diophantine approximation [1, Theorem 32].

Khinchin's Theorem. Let $\left(b_{n}\right)$ be a sequence of positive real numbers such that the sequence $\left(n b_{n}\right)$ is nonincreasing and the series $\sum_{n} b_{n}$ diverges. For almost all real numbers $x$, there are infinitely many integers $n$ such that $\operatorname{dist}(n x, \mathbb{Z})<b_{n}$.

We will also make use of the following lemma, which will be proved in the sequel.

Lemma 1. Let $\left(c_{n}\right)$ be a sequence of nonnegative real numbers going to zero. There exists a sequence of positive real numbers $\left(b_{n}\right)$ such that the sequence $\left(n b_{n}\right)$ is nonincreasing, $\sum_{n} b_{n}=+\infty$, and $\sum_{n} b_{n} c_{n}<+\infty$.

Let us prove Theorem 2 .

Replacing if necessary $a_{n}$ by $\inf _{k \geq n} a_{k}$, we can suppose that the sequence $\left(a_{n}\right)$ is nondecreasing. Applying the preceding lemma to the the sequence $c_{n}=1 / \sqrt{a_{n}}$, we obtain a sequence $\left(b_{n}\right)$ such that the sequence $\left(n b_{n}\right)$ is nonincreasing, $\sum_{n} b_{n}=+\infty$, and $\sum_{n} b_{n} / \sqrt{a_{n}}<+\infty$. The sequence $\left(b_{n}\right)$ tends to zero, and we can impose the additional requirement that $b_{n}<1 / 2$ for all $n$.

We consider the function $f_{1}$ defined on $\mathbb{R}$ by

$$
f_{1}(x)= \begin{cases}1 / \sqrt{a_{n}} & \text { if }|x-n| \leq b_{n} \text { for an integer } n \geq 1 \\ 0 & \text { if not. }\end{cases}
$$

This function is integrable, due to the last condition imposed on $\left(b_{n}\right)$.

By Khinchin's theorem, for almost all $x>0$, there exist pairs of positive integers $(n, k(n))$, with arbitrarily large $n$, such that

$$
|n x-k(n)| \leq b_{n} .
$$

Let us consider one fixed such $x$ in the interval $(0,1)$. We have $\lim _{n \rightarrow \infty} k(n)=+\infty$ and, since $\lim _{n \rightarrow+\infty} b_{n}=0$, we have $k(n) \leq n$ for all large enough $n$. For such an 
$n$, we have

$$
|n x-k(n)| \leq b_{k(n)} \quad \text { and hence } \quad f_{1}(n x)=\frac{1}{\sqrt{a_{k(n)}}} .
$$

(We used here the fact that the sequence $\left(b_{n}\right)$ is nonincreasing.) Thus, for arbitrarily large $n$, we have

$$
a_{n} f_{1}(n x)=\frac{a_{n}}{\sqrt{a_{k(n)}}} \geq \sqrt{a_{k(n)}} .
$$

(We used here the fact that the sequence $\left(a_{n}\right)$ is nondecreasing.) This proves that $\limsup _{n \rightarrow \infty} a_{n} f_{1}(n x)=+\infty$. This argument applies to almost all $x$ between 0 and 1.

For each integer $m \geq 1$, let us denote by $f_{m}$ the function $f_{m}(x)=f_{1}(x / m)$. This function $f_{m}$ is nonnegative and integrable on $\mathbb{R}$. It is locally a step function. For almost all $x$ between 0 and $m$, we have

$$
\limsup _{n \rightarrow \infty} a_{n} f_{m}(n x)=+\infty .
$$

From this, it is not difficult to construct a continuous and integrable function $f$ on $\mathbb{R}$ such that, for all $m>0$, there exists $A_{m}>0$ with $f \geq f_{m}$ on $\left[A_{m},+\infty\right.$ ). (For example, we can choose an increasing sequence of numbers $\left(A_{m}\right)$ such that

$$
\int_{A_{m}}^{+\infty} f_{1}(x)+f_{2}(x)+\cdots+f_{m}(x) \mathrm{d} x \leq \frac{1}{m^{2}}
$$

then we define $g=f_{1}+f_{2}+\cdots+f_{m}$ on the interval $\left[A_{m}, A_{m+1}\right)$. Since

$$
\sum_{m} \int_{A_{m}}^{A_{m+1}} f_{1}(x)+f_{2}(x)+\cdots+f_{m}(x) \mathrm{d} x<\infty,
$$

this function $g$ is integrable. Then we just have to find a continuous and integrable function $f$ which dominates $g$; this can be achieved since the function $g$ is locally a step function: choose $f$ to be zero on $(-\infty, 0]$ and continuous on $\mathbb{R}$ such that $g \leq f$ and, for all $m>0, \int_{m-1}^{m} f(x)-g(x) \mathrm{d} x \leq 1 / m^{2}$, so that $\int_{0}^{+\infty} f(x)-g(x) \mathrm{d} x<$ $+\infty$.)

For almost all $x \geq 0$, we have $\limsup _{n \rightarrow \infty} a_{n} f(n x)=+\infty$. A symmetrization procedure extends this property to almost all real numbers.

The first part of Theorem 2 is proved. The second part is a direct consequence. We consider the function $f$ constructed above, and we denote by $F$ the set of $x$ such that the sequence $\left(a_{n} f(n x)\right)$ is bounded. The set $\{n x \mid x \in F, n \in \mathbb{N}\}$ has zero measure. We modify the function $f$ on this set, choosing for example the value 1. The new function is integrable and satisfies, for all $x, \limsup _{n \rightarrow \infty} a_{n} f(n x)=$ $+\infty$.

Proof of Lemma 1. The sequence $\left(c_{n}\right)$ is given, and it goes to zero. We will construct by induction an increasing sequence of integers $\left(n_{k}\right)$ and a nonincreasing sequence of positive numbers $\left(d_{k}\right)$, and we will define $b_{n}=d_{k} / n$ for $n_{k-1} \leq n<n_{k}$. The numbers $d_{k}$ will be chosen so that $\sum_{i=n_{k-1}}^{n_{k}-1} b_{i}=1$; thus we require that

$$
d_{k}:=\left(\sum_{i=n_{k-1}}^{n_{k}-1} \frac{1}{i}\right)^{-1} .
$$


We start from $n_{0}=1$, and then we choose $n_{1}>n_{0}$ such that, for all $n \geq n_{1}$, $\left|c_{n}\right| \leq 1 / 2$. In the next step, we choose $n_{2}>n_{1}$ such that $d_{2} \leq d_{1}$ and, for all $n \geq n_{2},\left|c_{n}\right| \leq 1 / 4$.

More generally, if $n_{1}, n_{2}, \ldots, n_{k-1}$ have been constructed, we choose $n_{k}>n_{k-1}$ such that $d_{k} \leq d_{k-1}$ and, for all $n \geq n_{k},\left|c_{n}\right| \leq 2^{-k}$. (Of course, this is possible because $\lim _{n \rightarrow+\infty}\left(\sum_{i=n_{k-1}}^{n} \frac{1}{i}\right)^{-1}=0$.)

This defines the sequence $\left(b_{n}\right)$ by blocks. The sequence $\left(n b_{n}\right)$ is nonincreasing and, for all $k \geq 1$, we have

$$
\sum_{i=n_{k-1}}^{n_{k}-1} b_{i}=1 \text { and } \sum_{i=n_{k-1}}^{n_{k}-1} b_{i} c_{i} \leq 2^{1-k} .
$$

This guarantees that $\sum_{n} b_{n}=+\infty$ and $\sum_{n} b_{n} c_{n}<+\infty$. The lemma is proved.

About Remark 3. Dirichlet's lemma in Diophantine approximation (based on the pigeon-hole principle) concerns the particular case $b_{n}=1 / n$ in Khinchin's theorem and it gives a result for all $x$.

Lemma 2 (Dirichlet's Lemma). For all real numbers $x$, there exist infinitely many integers $n$ such that $\operatorname{dist}(n x, \mathbb{Z}) \leq \frac{1}{n}$.

Now, we justify Remark 3. We consider a nondecreasing sequence of positive real numbers $\left(a_{n}\right)$ such that

$$
\sum_{n} \frac{1}{n a_{n}}<+\infty
$$

We claim that there exists a sequence of positive real numbers $\left(b_{n}\right)$ such that

$$
b_{n} a_{n} \rightarrow+\infty \text { and } \sum \frac{b_{n}}{n}<+\infty
$$

Here is a proof of this claim: for each $k \geq 1$, there exists $n(k)$ such that

$$
\sum_{n \geq n(k)} \frac{1}{n a_{n}} \leq \frac{1}{k^{2}} \text {. }
$$

We have

$$
\sum_{n} \operatorname{card}\{k \mid n(k) \leq n\} \frac{1}{n a_{n}}=\sum_{k \geq 1} \sum_{n \geq n(k)} \frac{1}{n a_{n}}<+\infty,
$$

and we can define $b_{n}:=\operatorname{card}\{k \mid n(k) \leq n\} / a_{n}$.

Given this sequence $\left(b_{n}\right)$, we consider the function $f$ defined on $\mathbb{R}$ by

$$
f(x)= \begin{cases}b_{k} & \text { if }|x-k| \leq 1 / k, k \text { an integer, } k \geq 2, \\ 0 & \text { if not. }\end{cases}
$$

This function is integrable.

Using Dirichlet's lemma, we have the following: for each fixed $x$ in $(0,1)$, there exist pairs of positive integers $(n, k(n))$, with $n$ arbitrarily large, such that $\mid n x-$ $k(n) \mid \leq 1 / n$. We have $\lim _{n \rightarrow \infty} k(n)=+\infty$ and, for all large enough $n, k(n) \leq n$. Hence there exist infinitely many $n$ 's such that

$$
|n x-k(n)| \leq \frac{1}{k(n)} \quad \text { and so } \quad f(n x)=b_{k(n)} .
$$


For such an $n$, we have

$$
a_{n} f(n x)=a_{n} b_{k(n)} \geq a_{k(n)} b_{k(n)} .
$$

(We used here the fact that the sequence $\left(a_{n}\right)$ is nondecreasing.) This proves that $\limsup _{n \rightarrow \infty} a_{n} f(n x)=+\infty$. This result obtained for all numbers $x$ between 0 and 1 extends to all real numbers by the same argument as the one used in the proof of Theorem 2. We can also replace the local step function by a continuous one as we did before.

Theorem 1 answers a question asked by Aris Danilidis.

\section{REFERENCES}

[1] A. Ya. Khinchin, Continued Fractions, Dover, Mineola, NY, 1997; reprint of (trans. Scripta Technica, Inc.) University of Chicago Press, 1961; reprint of 3rd Russian ed., State Publishing House of Physical-Mathematical Literature, Moscow, 1961.

Laboratoire de Mathématiques et Physique Théorique,

Université François-Rabelais Tours, Fédération Denis Poisson - CNRS,

Parc de Grandmont, 37200 Tours, France

emmanuel.lesigne@lmpt.univ-tours.fr 